\title{
A GERŠGORIN INCLUSION SET FOR THE FIELD OF VALUES OF A FINITE MATRIX
}

\author{
CHARLES R. JOHNSON ${ }^{1}$
}

Abstract. An easily computed Geršgorin type inclusion set for the field of values of an $n$ by $n$ complex matrix is presented. Some functional properties of this inclusion set parallel those of the field of values, and illustrative examples are given.

1. Introduction. Let $M_{n}(C)$ denote the set of $n$ by $n$ matrices over the complex field. For $A=\left(a_{i j}\right) \in M_{n}(C)$, define

and let

$$
R_{i}(A)=\sum_{j=1, j \neq i}^{n}\left|a_{i j}\right| \text { and } C_{j}(A)=\sum_{i=1, i \neq j}^{n}\left|a_{i j}\right|
$$

$$
\begin{aligned}
& G_{r}(A)=\bigcup_{i=1}^{n}\left\{z:\left|z-a_{i i}\right| \leqq R_{i}(A)\right\}, \\
& G_{c}(A)=\bigcup_{j=1}^{n}\left\{z:\left|z-a_{j j}\right| \leqq C_{j}(A)\right\} .
\end{aligned}
$$

The well known theorem of Geršgorin [2] notes that the spectrum $\sigma(A)$ of $A$ is contained in $G_{r}(A) \cap G_{c}(A)$.

Denote the field of values of $A \in M_{n}(C)$ by

$$
F(A)=\left\{x A x^{*}: x \in C^{n}, x x^{*}=1\right\} .
$$

The bounded complex set $F(A)$ is convex, $\sigma(A) \subseteq F(A)$, and $F(A)$ is invariant under unitary similarities of $A$. In case $A$ is normal, $F(A)$ is the convex hull of $\sigma(A)$.

For a set $S$ in the complex plane, let $\operatorname{Co}(S)$ be its convex hull, let $g_{i}(A)=\left(R_{i}(A)+C_{i}(A)\right) / 2$ and define

$$
G(A)=\operatorname{Co}\left(\bigcup_{i=1}^{n}\left\{z:\left|z-a_{i i}\right| \leqq g_{i}(A)\right\}\right) .
$$

Received by the editors January 22, 1973.

AMS (MOS) subject classifications (1970). Primary 15A45, 15A63; Secondary 65F99.

Key words and phrases. Geršgorin set, field of values, subadditive set valued function, spectrum, eigenvalues, numerical radius.

1 This work was done while the author was a National Academy of Sciences-National Research Council Postdoctoral Research Associate at the National Bureau of Standards, Washington, D.C. 20234.

(c) American Mathematical Society 1973 
It is the goal of this note to present for $F(A)$ an analog of Geršgorin's theorem: $F(A) \subseteq G(A)$. Considered as set valued functions of a matrix argument $G$ and $F$ also share several functional properties.

By the sum of two sets $S_{1}+S_{2}$ we shall mean $\left\{x_{1}+x_{2}: x_{1} \in S_{1}, x_{2} \in S_{2}\right\}$ and let $R=\{z: \operatorname{Re}(z)>0\}$ denote the right complex half-plane.

2. Functional properties of $G$ and $F, \quad G$ and $F$ may be considered as functions from $M_{n}(C)$ into the class of convex subsets of the complex plane. As such they have many functional properties in common. We have already noted that $F(A)$ and $G(A)$ both contain $\sigma(A)$ and the first four of the following remarks note functional properties of $G$ which are well known for $F$.

REMARK 1. For any complex number $\alpha, G(A-\alpha I)=G(A)+\{-\alpha\}$ and $G(\alpha A)=\alpha G(A)$.

REMARK 2. If $A_{0} \in M_{k}(C)$ is a principal submatrix of $A \in M_{n}(C)$, $k \leqq n$, then $G\left(A_{0}\right) \subseteq G(A)$.

Proof. This follows from the observation that if $A_{0}$ is determined by the indices $i_{1}, \cdots, i_{k}$, then $g_{j}\left(A_{0}\right) \leqq g_{i_{j}}(A), j=1, \cdots, k$.

RemarK 3. $G$ is subadditive. That is for $A, B \in M_{n}(C), G(A+B) \subseteq$ $G(A)+G(B)$.

Proof. Let $A=\left(a_{i j}\right), B=\left(b_{i j}\right)$. Because of the triangle $g_{i}(A+B) \leqq$ $g_{i}(A)+g_{i}(B)$ for all $i=1, \cdots, n$. It follows that

$$
\begin{aligned}
&\left\{z:\left|z-\left(a_{i i}+b_{i i}\right)\right| \leqq g_{i}(A+B)\right\} \\
& \subseteq\left\{z:\left|z-a_{i i}\right| \leqq g_{i}(A)\right\}+\left\{z:\left|z-b_{i i}\right| \leqq g_{i}(B)\right\}
\end{aligned}
$$

which implies $G(A+B) \subseteq G(A)+G(B)$.

REMARK 4. If $A \in M_{n}(C)$ is diagonal, then $F(A)=G(A)$.

REMARK 5. Unlike $F, G(A)$ is not invariant under unitary similarities of $A$.

3. Main result. We next show that $G(A)$ is also an upper estimate for $F(A)$ for all $A \in M_{n}(C)$.

LEMMA 1. If $G(A) \subseteq R$, then $F(A) \subseteq R$.

Proof. Let $A=\left(a_{i j}\right)$; let the Hermitian part of $A$ be $\left(b_{i j}\right)=B=$ $\left(A+A^{*}\right) / 2$; and suppose $G(A) \subseteq R$ which means $\operatorname{Re}\left(a_{i i}\right)>g_{i}(A)$. Since $R_{i}\left(A^{*}\right)=C_{i}(A)$ and because of the triangle inequality, $R_{i}(B) \leqq g_{i}(A)$ and we have $b_{i i}=\operatorname{Re}\left(a_{i i}\right)>g_{i}(A) \geqq R_{i}(B)$. Then since $\sigma(B) \subseteq G_{r}(B)$ by Geršgorin's theorem and since $G_{r}(B) \subseteq R$ because $b_{i i}>R_{i}(B)$ we obtain that $\sigma(B) \subseteq R$. But since $B$ is Hermitian $F(B)=\operatorname{Co}(\sigma(B))$ and thus $F(B) \subseteq R$. Now $A=B+C$ where $C=\left(A-A^{*}\right) / 2$. Since $F(C)$ is pure imaginary $F(B)+F(C) \subseteq R$ and by the subadditivity of $F, F(A) \subseteq R$ as was to be shown. 
Lemma 2. If $0 \notin G(A)$, then $0 \notin F(A)$.

Proof. Suppose $0 \notin G(A)$. Since $G(A)$ is convex, there is a $\theta, 0 \leqq \theta<2 \pi$, such that $G\left(e^{i \theta} A\right)=e^{i \theta} G(A) \subseteq R$. By Lemma 1 this implies $F\left(e^{i \theta} A\right) \subseteq R$, and since $F(A)=e^{-i \theta} F\left(e^{i \theta} A\right)$, it follows that $0 \notin F(A)$.

THEOREM. For all $A \in M_{n}(C), F(A) \subseteq G(A)$.

Proof. Suppose $\alpha \in F(A)$. Then $0 \in F(A-\alpha I)$ and by the contrapositive of Lemma 2, $0 \in G(A-\alpha I)$. Because of Remark 1, we may conclude that $\alpha \in G(A)$. Thus $F(A) \subseteq G(A)$ which completes the proof.

If we denote the numerical radius, $\max _{\alpha \in F(A)}|\alpha|$, of $A \in M_{n}(C)$ by $r(A)$, then we may obtain an estimate for $r(A)$ by the preceding theorem.

Corollary. For $A \in M_{n}(C)$,

$$
r(A) \leqq \max _{i}\left(\left|a_{i i}\right|+g_{i}(A)\right)=\max _{i}\left(\sum_{j=1}^{n} \frac{\left|a_{i j}\right|+\left|a_{j i}\right|}{2}\right) .
$$

Proof. Because of the theorem, it merely suffices to note that $\max _{\alpha \in G(A)}|\alpha|=\max _{i}\left(\left|a_{i i}\right|+g_{i}(A)\right)$ which is valid since $G(A)$ is the convex hull of a closed set whose largest element in absolute value is

$$
\max _{i}\left(\left|a_{i i}\right|+g_{i}(A)\right) \text {. }
$$

4. Examples and further remarks. Our first example shows that $G(A)$ gives the most economical general estimate of its type for $F(A)$, and the third shows how much of an overestimate $G(A)$ can be in an extreme case. We then give an application which is a sufficient condition for $F(A)$ to be a circle (with interior).

EXAMPLE 1. Let $A=\left(\begin{array}{ll}0 & 2 \\ 0 & 0\end{array}\right)$. It then may be computed that $F(A)=G(A)$ which is the unit circle.

Suppose $s(x, y)$ is a function of the two nonnegative real variables $x$ and $y$, and let $A=\left(a_{i j}\right)$ be an arbitrary element of $M_{n}(C)$. Let $s_{i}=$ $s\left(R_{i}(A), C_{i}(A)\right)$ and define

$$
G_{s}(A)=\operatorname{Co}\left(\bigcup_{i=1}^{n}\left\{z:\left|z-a_{i i}\right| \leqq s_{i}\right\}\right) .
$$

If $F(A) \subseteq G_{s}(A)$ for all $A$, it then follows from Example 1 that $G(A) \subseteq$ $G_{s}(A)$ and $s(x, y) \geqq(x+y) / 2$, and $G(A)$ is the best upper estimate of this type.

EXAMPLE 2. That the geometric mean does not provide an upper estimate for $F(A)$ is shown by letting $A=\left(\begin{array}{ll}2 & 3 \\ 1 & 2\end{array}\right)$ and $s(x, y)=(x y)^{1 / 2}$. Then $G_{s}(A)$ is the circle about 2 of radius $3^{1 / 2}$, but it is easily seen that $0 \in F(A)$ so that $F(A) \nsubseteq G_{s}(A)$. 
EXAmple 3. Let

$$
A=\left[\begin{array}{cccc}
0 & 1 & \cdots & 1 \\
1 & 0 & \cdots & 0 \\
\cdot & \cdot & & \\
\cdot & \cdot & 0 & \\
\cdot & \cdot & & \\
1 & 0 & &
\end{array}\right] \in M_{n}(C)
$$

Then $F(A)$ may be computed to be $\left[-(n-1)^{1 / 2},(n-1)^{1 / 2}\right]$ with $r(A)=$ $(n-1)^{1 / 2}$. Since $G(A)$ is the circle about the origin of radius $n-1, G(A)$ is a heavy overestimate in this extreme case. However, if

$$
B=\left[\begin{array}{cccc}
0 & 1 & \cdots & 1 \\
1 & 0 & & 1 \\
\cdot & \cdot & & \\
\cdot & 1 & \cdot & \\
\cdot & & \cdot & \\
1 & & & 0
\end{array}\right] \in M_{n}(C),
$$

$G(B)$ is again the circle of radius $n-1$ about the origin. But $F(B)=$ $[1-n, n-1]$ with $r(B)=n-1$.

Remark 6. It is easy to see [1] that any $A \in M_{n}(C)$ may be unitarily transformed to $\left(b_{i j}\right)=B=U^{*} A U$ with $b_{i i}=T_{r}(A) / n, i=1, \cdots, n$. Suppose without loss of generality that $g_{1}(B) \geqq g_{i}(B), i=2, \cdots, n$, also. Then if row 1 and column 1 of $B$ contain at most one nonzero entry besides $b_{11}$ (for instance $b_{1 n}$ ), $F(A)$ is a circle about $T_{r}(A) / n$ (of radius $g_{1}(B)=$ $\left.\left|b_{1 n}\right| / 2\right)$. Let $a=T_{r}(A) / n$ and this follows since

$$
\begin{aligned}
G\left(\left[\begin{array}{cc}
a & b_{1 n} \\
0 & a
\end{array}\right]\right) & =F\left(\left[\begin{array}{cc}
a & b_{1 n} \\
0 & a
\end{array}\right]\right) \subseteq F(B) \subseteq\left\{z:|z-a| \leqq g_{1}(B)\right\} \\
& =G\left(\left[\begin{array}{cc}
a & b_{1 n} \\
0 & a
\end{array}\right]\right)
\end{aligned}
$$

and $F(A)=F(B)$.

\section{REFERENCES}

1. W. V. Parker, Sets of complex numbers associated with a matrix, Duke Math. J. 15 (1948), 711-715. MR 10, 230.

2. S. Geršgorin, Uber die Abgrenzung der Eigenwerte einer Matrix, Izv. Akad. Nauk SSSR 7 (1931), 749-754.

Applied Mathematics Division, National Bureau of Standards, Washington, D.C. 20234 